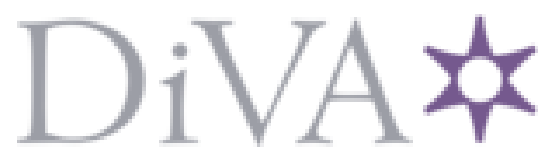

http://www.diva-portal.org

This is the published version of a paper presented at 2008 International Conference on Advanced Infocomm Technology, ICAIT '08. Shenzhen. 29 July 2008 - 31 July 2008.

Citation for the original published paper:

Du, J., Signell, S. (2008)

Pulse Shape Adaptivity in OFDM/OQAM Systems.

In: 2008 International Conference on Advanced Infocomm Technology, ICAIT '08 Association for Computing Machinery (ACM)

http://dx.doi.org/10.1145/1509315.1509446

N.B. When citing this work, cite the original published paper.

Permanent link to this version:

http://urn.kb.se/resolve?urn=urn:nbn:se:kth:diva-31817 


\title{
Pulse Shape Adaptivity in OFDM/OQAM Systems
}

\author{
Jinfeng Du, and Svante Signell, Senior Member, IEEE \\ Department of Communication Systems \\ School of Information and Communication Technology \\ KTH - Royal Institute of Technology, SE-16440 Kista, Stockholm, Sweden \\ \{jinfeng, srs\}@kth.se
}

\begin{abstract}
Adaptation is crucial to realise high data rate transmission in multicarrier communication systems over dispersive channels. Apart from rate/power adaptation enabled by orthogonal frequency division multiplexing (OFDM), OFDM/offset QAM (OFDM/OQAM) systems provide possibility to adjust pulse shapes regarding to the channel characteristics. In this paper we discuss and evaluate pulse shape adaptivity in OFDM/OQAM systems with focus on the extended Gaussian functions (EGF) which have been shown to be good candidates for pulse shape adaptation. By investigating the time frequency dispersion robustness and carrier frequency offset sensitivity, both analysis and simulation results show that pulse shape adaptation with respect to the channel state information can improve the system performance.
\end{abstract}

\section{INTRODUCTION}

Multicarrier communication technologies are promising candidates to realize high data rate transmission in Beyond 3G and further wireless systems where the channel is mostly doubly dispersive. Contrary to the classic OFDM system using a cyclic prefix (CP-OFDM) to combat time dispersion, OFDM/OQAM [1, 2] which utilises well designed pulse shapes and/or system lattice can achieve smaller ISI/ICI without using the cyclic prefix. Performance evaluation of OFDM/OQAM has already illustrated promising advantage [3, 4] and it has already been introduced in the TIA's Digital Radio Technical Standards [5] and been considered in WRAN (IEEE 802.22) [4], where the robustness to frequency dispersion is not taken into account.

Among other famous pulse functions, the extended Gaussian function (EGF) [6] is well known for its localisation variation in the time-frequency plane. Therefore it plays a vital role in the OFDM/OQAM pulse shape adaptation in the following discussion. Besides, adjustment of other parameters, such as FFT size, sampling frequency, etc., will also change the overall performance largely. The purpose of

Permission to make digital or hard copies of all or part of this work for personal or classroom use is granted without fee provided that copies are not made or distributed for profit or commercial advantage and that copies bear this notice and the full citation on the first page. To copy otherwise, or republish, to post on servers or to redistribute to lists, requires prior specific permission and/or a fee.

ICAIT'08, July-Aug, 2008, Shenzhen, China.

Copyright 2008 ACM 978-1-60558-088-3...\$5.00. this paper is to investigate and evaluate pulse shape adaptivity in OFDM/OQAM systems to see how it can affect the performance over dispersive channels.

The rest of this paper is organized as follows. Section 2 presents the system model and Section 3 introduces two time frequency localisation (TFL) parameters and their relationship to system performance with respect to channel realisations are discussed. In Section 4 the sensitivity of OFDM/OQAM systems to carrier frequency offset are discussed. Uncoded bit error rate simulation results with various parameter adaptation over time dispersion channels are presented in Section 5 and conclusions are drawn in Section 6.

\section{SYSTEM MODEL}

The transmitted signal in CP-OFDM and OFDM/OQAM systems can be written in the following analytic form

$$
s(t)=\sum_{n=-\infty}^{+\infty} \sum_{m=0}^{N-1} a_{m, n} g_{m, n}(t)
$$

where $a_{m, n}(n \in \mathbb{Z}, m=0,1, \ldots, N-1)$ denotes the symbol conveyed by the sub-carrier of index $m$ during the symbol time of index $n$, and $g_{m, n}(t)$ represents the synthesis basis which is obtained by time-frequency translation of the prototype function $g(t)$. In CP-OFDM systems

$$
g_{m, n}(t)=e^{j 2 \pi m F t} g\left(t-n\left(T+T_{c p}\right)\right), \quad T F=1
$$

where $T$ and $F$ are the symbol duration and inter-carrier frequency spacing respectively, $a_{m, n}$ are complex valued symbols and $g(t)$ is the rectangular function. In OFDM/OQAM systems

$$
g_{m, n}(t)=e^{j(m+n) \pi / 2} e^{j 2 \pi m \nu_{0} t} g\left(t-n \tau_{0}\right), \quad \nu_{0} \tau_{0}=1 / 2
$$

where the real part and imaginary part of the complex symbol $a_{m, n}$ are transmitted separately with symbol duration $\tau_{0}$ and inter-carrier spacing $\nu_{0}$ respectively. Hence OFDM/OQAM systems transmit at half symbol rate but with doubly density compared with CP-OFDM systems if the length of cyclic prefix equals to zero.

Two kinds of realizations of pulse shaping OFDM/OQAM systems are of practical interest as they are very easy implement in classic OFDM systems. One can either set $\nu_{0}=F$ and shorten the symbol duration, or set $\tau_{0}=T$ and double the number of sub-carriers. We use the former approach in 
this paper via the efficient implementation method derived in [7].

After passing through the doubly dispersive channel, the received signal (noise is omitted here for simplicity) can be written as

$$
\begin{aligned}
r(t) & =\int h(t, \tau) s(t-\tau) d \tau=\iint H(\nu, \tau) s(t-\tau) e^{j 2 \pi \nu t} d \nu d \tau \\
& =\iint H(\nu, \tau) \sum_{m, n} a_{m, n} g_{m, n}(t-\tau) e^{j 2 \pi \nu t} d \nu d \tau
\end{aligned}
$$

where $h(t, \tau)$ is the channel impulse response and $H(\nu, \tau)$ is its Fourier Transform with respect to $t$. Without loss of generality, we assume symbol $a_{0,0}$ is going to be detected,

$$
\begin{gathered}
\hat{a}_{0,0}=<r(t), g_{0,0}(t)>=\int r(t) g_{0,0}^{*}(t) d t \\
=\iiint H(\nu, \tau) \sum_{m, n} a_{m, n} g_{m, n}(t-\tau) g_{0,0}^{*}(t) e^{j 2 \pi \nu t} d t d \nu d \tau
\end{gathered}
$$

Define the ambiguity function ${ }^{1}$ as

$$
A_{g}(\tau, \nu)=\int_{\mathbb{R}} e^{-j 2 \pi \nu t} g(t+\tau / 2) g^{*}(t-\tau / 2) d t
$$

then for OFDM/OQAM (5) can be rewritten as (8), shown on the top of next page. Under the assumption of wide sense stationary uncorrelated scattering (WSSUS) channel, we have

$$
E\left\{H(\nu, \tau) H^{*}\left(\nu^{\prime}, \tau^{\prime}\right)\right\}=S_{h}(\tau, \nu) \delta\left(\tau-\tau^{\prime}, \nu-\nu^{\prime}\right)
$$

where $E\{\}$ is the expectation operator and $S_{h}(\tau, \nu)$ is the channel scattering function. Assume all the transmitted symbols are independent with uniform energy, i.e., $E\left\{a_{m, n} a_{m^{\prime}}^{*}\right.$ $\delta_{m m^{\prime}} \delta n n^{\prime}$, and apply the WSSUS assumption (6), the energy of the desired signal part $S$ and the interference part $I$ in (8) can be written as

$$
\begin{aligned}
& E_{S}=E\left\{S S^{*}\right\}=\iint S_{h}(\tau, \nu)\left|A_{g}(\tau, \nu)\right|^{2} d \nu d \tau \\
& E_{I}=\sum_{(m, n) \neq(0,0)} \iint S_{h}(\tau, \nu)\left|A_{g}\left(n \tau_{0}+\tau, m \nu_{0}+\nu\right)\right|^{2} d \nu d \tau
\end{aligned}
$$

which are the same as, at least on the analogy of, the energy expressions derived for pulse shape multicarrier systems [10] and for hexagonal multicarrier systems [9]. Different optimisation methods regarding maximising desired signal energy $E_{S}$ [11], or minimising interference $E_{I}[10,9]$, or maximising the signal to interference ratio $E_{S} / E_{I}[10]$ are considered. However analytical solutions only exist for some special cases and therefore numerical solutions are used for general cases.

\section{TIME FREQUENCY LOCALISATION PA- RAMETERS}

For different channels, the optimal pulse shape is normally different. A widely used parameter to measure the time frequency localization of the pulse shape is the Heisenberg parameter [2] $\xi=\frac{1}{4 \pi \Delta t \Delta f} \leq 1$ with its maximum achieved by the Gaussian function $g_{\alpha}(t)=(2 \alpha)^{1 / 4} e^{-\pi \alpha t^{2}}, \alpha>0 . \Delta t$ is the mass moment of inertia of the prototype function in time and $\Delta f$ in frequency, which indicates how the energy (mass) of the prototype function spreads over the time and

${ }^{1}$ There is another definition for the ambiguity function, which differs by a phase shift.

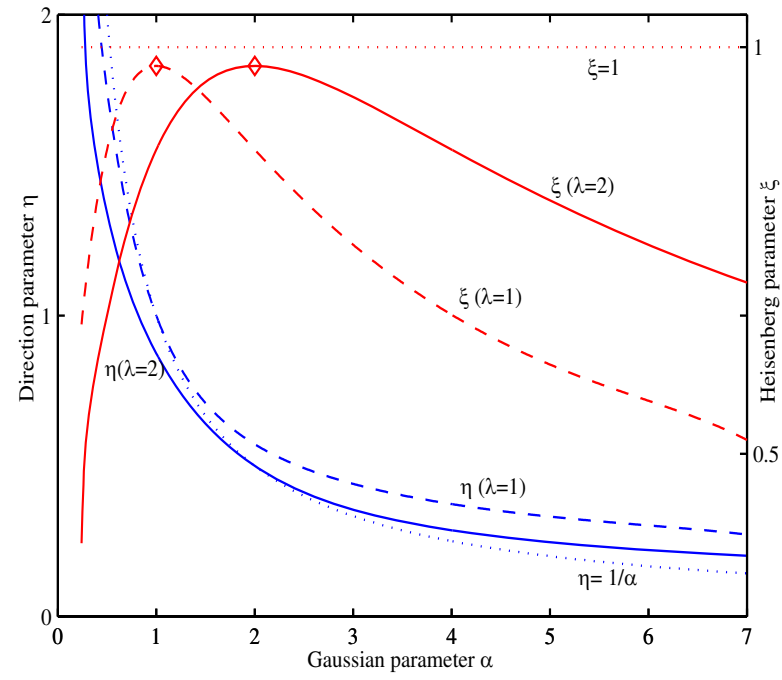

Figure 1: TFL parameters $(\xi, \eta)$ for EGF with $\lambda=1$ (dashed line), $\lambda=2$ (solid line). TFL for the Gaussian function (dotted line) is plotted as reference.

frequency plane. The larger $\xi$ is, the smaller space the pulse shape occupies in the T-F plane.

$$
\begin{cases}(\Delta t)^{2} & =\int_{\mathbb{R}} t^{2}|g(t)|^{2} d t \\ (\Delta f)^{2} & =\int_{\mathbb{R}} f^{2}|G(f)|^{2} d f\end{cases}
$$

Here $g(t)$ is assumed to be origin-centered with unity energy [8] for simple expressions.

$\left.{ }^{\prime}\right\}$ Fn order to know how the pulse shape spreads over the T$\mathrm{F}$ plane, we define the Direction parameter $\eta=\frac{\Delta t}{\Delta f}$. For EGF functions with $\frac{\tau_{0}}{T}=\sqrt{\frac{1}{2 \lambda}}$ and $\frac{\nu_{0}}{F}=\sqrt{\frac{\lambda}{2}}$, where $\lambda>0$ is a constant scaling factor, the variation of $\xi$ and $\eta$ with (7)espect to $\alpha$ for EGF functions with $\lambda=1\left(\frac{\tau_{0}}{T}=\frac{\nu_{0}}{F}=\right.$ $\left.\frac{\sqrt{2}}{2}\right)$ and $\lambda=2\left(\frac{\tau_{0}}{T}=\frac{1}{2}, \frac{\nu_{0}}{F}=1\right)$ is shown in Fig. 1, in which $\tau_{0}$ and $\nu_{0}$ are normalised by $T$ and $F$ respectively for convenience. Compared to the case with $\frac{\tau_{0}}{T}=\frac{\nu_{0}}{F}=\frac{\sqrt{2}}{2}$, the EGF function with $\frac{\tau_{0}}{T}=\frac{1}{2}$ have larger variation of $\eta$ and better stability of $\xi$, which makes it more suitable for pulse shape adaptation. The Gaussian function $g_{\alpha}(t)$ which has $\eta=1$, will not be taken into consideration since it will introduce large reconstruction distortion as we will see later.

To maximise the immunity to delay and frequency dispersion, the optimum pulse shape should have the same shape as the channel itself $[2,11]$, namely,

$$
\frac{\Delta t}{\Delta f} \approx \frac{\tau_{\mathrm{RMS}}}{f_{D}} \approx \frac{T}{F}
$$

where $\tau_{\mathrm{RMS}}$ is the root-mean-square (RMS) delay spread and $f_{D}$ is the maximum Doppler shift. If the value of $\eta$ is calculated with normalised $\tau_{0}$ and $\nu_{0}$ (by $T$ and $F$ respectively) as in Fig. 1, (10) can be rewritten as

$$
\eta(\alpha) \approx \frac{\tau_{\mathrm{RMS}} / T}{f_{D} / F}=\frac{\tau_{\mathrm{RMS}}}{f_{D}}\left(\frac{F_{s}}{N}\right)^{2}
$$




$$
\begin{aligned}
& \hat{a}_{0,0}=\sum_{m, n} a_{m, n} j^{m+n} \iint H(\nu, \tau) A_{g}\left(n \tau_{0}+\tau, m \nu_{0}+\nu\right) e^{j \pi\left(n \tau_{0}+\tau\right)\left(m \nu_{0}+\nu\right)} d \nu d \tau \\
& =\underbrace{a_{0,0} \iint H(\nu, \tau) A_{g}(\tau, \nu) e^{j \pi \tau \nu} d \nu d \tau}_{\text {Signal } S}+\underbrace{\sum_{(m, n) \neq(0,0)} a_{m, n} j^{m+n} \iint H(\nu, \tau) A_{g}\left(n \tau_{0}+\tau, m \nu_{0}+\nu\right) e^{j \pi\left(n \tau_{0}+\tau\right)\left(m \nu_{0}+\nu\right)} d \nu d \tau}_{\text {Interference } I}
\end{aligned}
$$

Therefore, for each specific channel realisation (i.e. $\frac{\tau_{\mathrm{RMS}}}{f_{D}}$ is determined), the performance against delay and Doppler dispersion depends on the bandwidth $F$ and the direction parameter $\eta$. We can adjust these two parameters to improve the system performance. When the sampling frequency $F_{s}$ is fixed in some instance, the FFT size $N$ will be subject to adaptation since $F=\frac{F_{s}}{N}$.

\section{ORTHOGONALITY PARAMETER $\gamma^{2}$ AND FREQUENCY OFFSET SENSITIVITY}

Define the orthogonality parameter for different pulse shapes as

$$
\gamma^{2}=E\left\{\left|\tilde{a}_{m, n}-a_{m, n}\right|^{2}\right\}
$$

where $a_{m, n}$ is the transmitted symbol, $\tilde{a}_{m, n}$ is the reconstructed signal. $\gamma^{2}$ can also be used to indicate the distortion power introduced by non-perfect reconstruction through an ideal channel $(r(t)=s(t))$, see Table 1. CP-OFDM and OFDM/OQAM with the half cosine function can achieve perfect reconstruction in the absence of a channel as the level of distortion power reaches the resolution limit of a double precision number $\left(\approx 10^{-15}\right)$. OFDM/OQAM with the EGF pulse shape introduce limited distortion due pulse shape truncation, and the distortion introduced by the Gaussian pulse is very significant due to lack of orthogonality.

Table 1: Distortion power after reconstruction

\begin{tabular}{|c|c|c|c|c|c|}
\hline pulse & OFDM & $\begin{array}{c}\text { half- } \\
\text { cosine }\end{array}$ & $\begin{array}{c}\text { Gaussian } \\
\alpha=1 \mid \alpha=2\end{array}$ & $\begin{array}{c}\text { EGF } \\
\alpha=1\end{array}$ & $\begin{array}{c}\text { EGF } \\
\alpha=2\end{array}$ \\
\hline$\gamma^{2}[\mathrm{~dB}]$ & -314 & -309 & $-11 \mid-22$ & -96 & -178 \\
\hline
\end{tabular}

Assume each block of data consists of $N_{r}$ frames and each frame contains $N$ data symbols in OFDM/OQAM and $N+$ $N_{c p}$ symbols in CP-OFDM respectively, with $N_{c p}$ denotes the number of cyclic prefix symbols inserted. Based on previous work in [12] and take the length of data block into consideration, it can be shown that in CP-OFDM the distortion power $\gamma^{2}$ introduced by carrier frequency offset $f_{\Delta}$ through an ideal channel (with only frequency offset added) can be written as

$$
\gamma_{O F D M}^{2}=\frac{4}{3}\left(\pi N N_{r} \frac{f_{\Delta}}{F_{s}}\left(1+\frac{N_{c p}}{N}\right)\right)^{2}
$$

where $N$ is FFT size, $\frac{f_{\Delta}}{F_{S}}$ is the normalised frequency offset. The number of frames $N_{r}$ per block appears since the phase shift caused by carrier frequency offset $f_{\Delta}$ accumulates as the length of data block increases, and therefore increase the distortion power. General expression for OFDM/OQAM with different pulse shapes has a similar form as for $\mathrm{CP}$ OFDM

$$
\gamma_{O Q A M}^{2}=\beta_{g} \frac{4}{3}\left(\pi N N_{r} \frac{f_{\Delta}}{F_{s}}\right)^{2}
$$

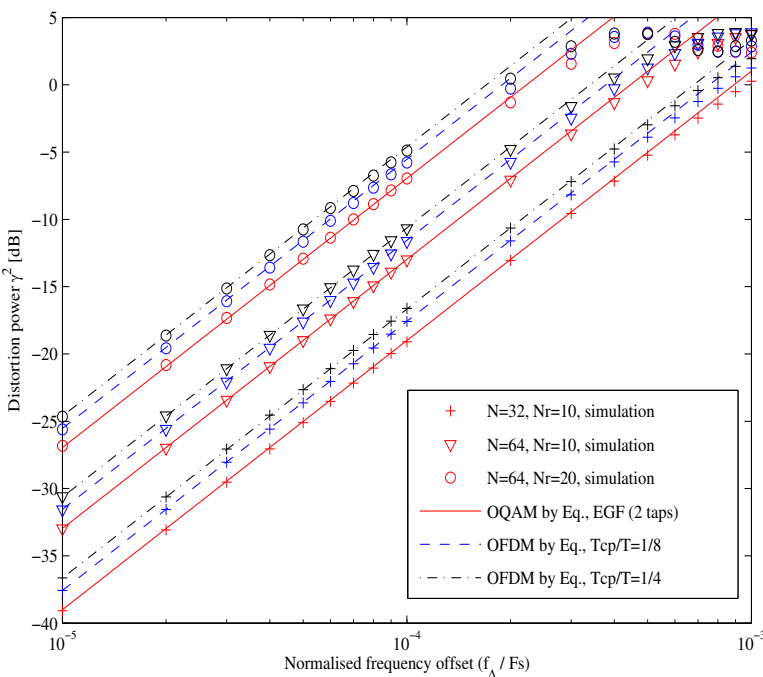

Figure 2: Frequency offset robustness for CPOFDM and OFDM/OQAM (EGF 2 taps) systems, 4QAM.

where $\beta_{g}>0$ is a scaling factor related to the pulse shape $g(t)$ and can be determined by numerical methods. The number of taps in the pulse shape filterbank will affect the value of $\beta_{g}$ since it will increase the length of the data block and therefore the phase shift, if the number of taps used is larger than 1. Therefore a trade off between orthogonality and frequency offset sensitivity has to be sort to achieve small $\beta_{g}$. Both simulation results (markers only) and curves by (13) and (14) are shown in Fig. 2. When the same system parameters are used, OFDM/OQAM always outperforms CP-OFDM by $0.9 \mathrm{~dB}$ to $2.3 \mathrm{~dB}$, in which about $0.5 \mathrm{~dB}$ $\left(\frac{T_{c p}}{T}=\frac{1}{16}\right)$ to $1.9 \mathrm{~dB}\left(\frac{T_{c p}}{T}=\frac{1}{4}\right)$ comes from not using the cyclic prefix.

\section{SIMULATION RESULTS}

In this section we present uncoded bit error rate (BER) simulation results carried out on the Matlab/Octave Simulation Workbench for Software Defined Radio [13]. A Monte Carlo-based WSSUS channel model [14] for doubly dispersive channels is extended and used. Assume that the time and frequency dispersive channel has $Q$ resolvable paths $h_{q}, q=0,1,2, \ldots, Q-1$, each with time spread $\tau_{q}$, Maximum Doppler shift $f_{q}$, power amplitude $\beta_{q}$ and random phase shift $\varphi_{q} \cdot \tau_{d}=\max _{i, j}\left|\tau_{i}-\tau_{j}\right|$ is defined as the delay spread and $B_{d}=2 f_{D}$ as the Doppler spread, where $f_{D}=\max _{q}\left(f_{q}\right)$ is the maximum Doppler frequency shift. With assumption of exponential delay power profile and U-shape Doppler power 
spectrum, we have

$$
S_{h}(\tau, \nu)=\frac{e^{-\frac{|\tau|}{\tau_{\mathrm{RMS}}}}}{\tau_{\mathrm{RMS}}} \frac{1}{\pi f_{D} \sqrt{1-\left(\frac{\nu}{f_{D}}\right)^{2}}}
$$

where $\tau_{\text {RMS }}$ is the RMS delay spread. Two time dispersive channels and one doubly dispersive channel are used in the following simulation, with the channel parameters listed in Table 2. For a carrier frequency $f_{c}=2.5 \mathrm{GHz}$, Doppler spread $B_{d}=2 f_{D}=700 \mathrm{~Hz}$ is equivalent to a moving speed of $157.5 \mathrm{~km} / \mathrm{h}$.

Table 2: Channel parameters

\begin{tabular}{|c|c|c|c|c|c|}
\hline channel & $\tau \in[\mathrm{ns}]$ & $\tau_{d}[\mathrm{~ns}]$ & $\tau_{\mathrm{RMS}}[\mathrm{ns}]$ & $B_{d}[\mathrm{~Hz}]$ & \#taps \\
\hline $\mathrm{A}$ & {$[0,4167]$} & 4167 & 1042 & 0 & $<10$ \\
\hline $\mathrm{B}$ & {$[-1042,3125]$} & 4167 & 1402 & 0 & $<10$ \\
\hline $\mathrm{C}$ & {$[0,4167]$} & 4167 & 1042 & 700 & $<10$ \\
\hline
\end{tabular}

In OFDM/OQAM systems each component filter has maximum 4 taps and a cyclic prefix with length $\frac{T_{c p}}{T}=\frac{1}{8}$ is used in the CP-OFDM system, unless mentioned otherwise. Frequency separation $F=15 k H z$ is used for both CP-OFDM and OFDM/OQAM, and $N_{r}=10$ frames are packed in one block and transmitted through tapped delay line channels. Each block contains one pilot frame for channel estimation and one-tap frequency domain equaliser (FDE) is used together with a normal AWGN symbol detector. In OFDM/OQAM systems, EGF with 4-tap filterbank and halfcosine with 1-tap filterbank are used. The Gaussian parameter $\alpha$ in EGF is chosen via numerical solution by maximising the signal power (denoted as $E_{S}$ ), or by minimising the interference power (denoted as $E I$ ). The lower bound $\alpha=0.5$ in EGF functions are chosen for reference.

Fig. 3 and Fig. 4 illustrates the BER performance of uncoded transmission for OFDM/OQAM through time dispersive channels. When channel A is used, the distortion caused by time dispersion is fully removed by cyclic prefix in CPOFDM and reduced by pulse shapes in OFDM/OQAM systems. The moderate gain of OFDM/OQAM compared with CP-OFDM in low SNR region mainly comes from the energy saved by not using the cyclic prefix $\left(0.51 \mathrm{~dB}\right.$ for $\left.\frac{N_{c p}}{N}=\frac{1}{8}\right)$. When channel B is used, OFDM/OQAM outperforms CPOFDM as the interference from "early" arrived paths cannot be removed by the cyclic prefix. Besides, a moderate spectral efficiency gain is achieved in OFDM/OQAM by not using the cyclic prefix. It is a little bit surprising that the performance of EGF with minimised interference power $(E I)$ performs worse than EGF with maximised signal power $\left(E_{S}\right)$, as you can see in Fig. 3 and Fig. 4. One possible reason is that the minimisation of interference power is based on the assumption of perfect equalisation, while it is not the case in our implementation with a one-tap FDE. Since channel A and channel B are purely time dispersive, a pulse with larger support in time domain will satisfy the requirement stated in (11), which means a smaller value of $\alpha$ for EGF functions. Therefore EGF with $\alpha=0.5$ Empirical observation shows that ${ }^{2}$ performance the best among different

${ }^{2} \alpha \in[0.5,7.5]$ for EGF functions will give a good trade off between time frequency localisation and orthogonality.

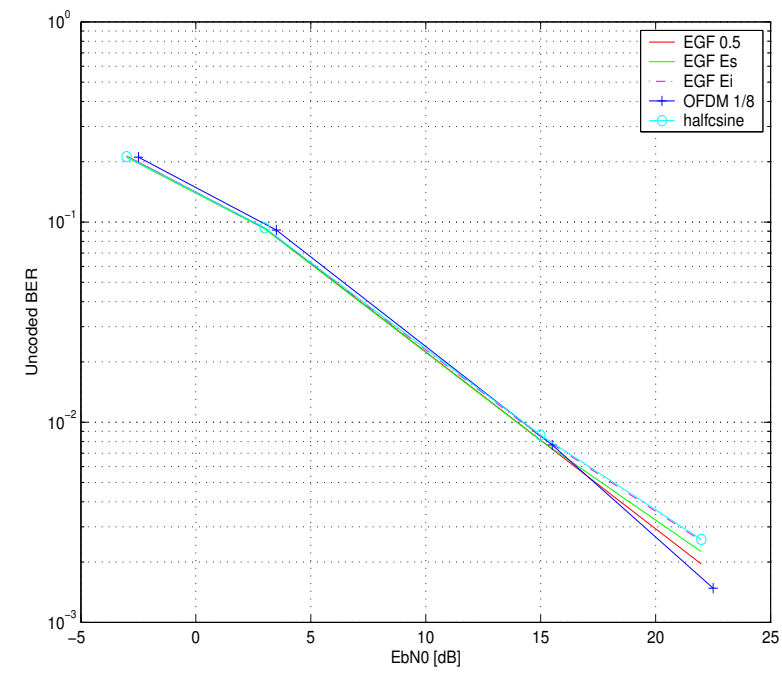

Figure 3: BER vs. SNR over channel A with $F=15 k H z$ for OFDM $\left(N_{c p}=\frac{N}{8}\right)$ and OFDM/OQAM with EGF (4 taps) and halfcosine (1 taps), 4QAM.

pulse shapes in OFDM/OQAM.

The uncoded BER performance over doubly dispersive channels are shown in Fig. 5. The performance degradation due to channel variation are very significant in all the systems, while OFDM/OQAM with different pulse shapes all outperform CP-OFDM. However, the difference between pulse shapes is not resolvable. A more powerful detector, such as minimum mean square error (MMSE) detector with successive interference cancellation [9], are needed to exploit the benefit of higher signal to interference ratio.

\section{CONCLUSIONS}

The performance of pulse shape adaptation in OFDM/OQAM systems over dispersive channels has been discussed and evaluated by investigating the time frequency dispersion robustness, carrier frequency offset immunity, and sensitivity to parameter variation. Both analysis and simulation results show that pulse shape adaptation with respect to the channel state information can actually improve the system performance. As the effect of $\alpha$ in EGF functions on OFDM/OQAM performance turns out to be not significant and therefore only an approximate value is enough. Since $\eta(\alpha) \propto \frac{1}{\alpha}$, reasonable approximation can be made in searching of the proper value of $\alpha$. With the help of the Gaussian parameter $\alpha$ for EGF functions, pulse shape adaptation can be easily realised.

\section{ACKNOWLEDGMENTS}

This work was supported in part by Wireless@KTH.

\section{REFERENCES}

[1] R. W. Chang, "Synthesis of Band-Limited Orthogonal Signals for Multi-carrier Data Transmission," Bell. Syst. Tech. J., vol. 45, pp. 1775-1796, Dec. 1966.

[2] B. le Floch, M. Alard and C. Berrou, "Coded Orthogonal Frequency Division Multiplex," Proceedings of the IEEE, vol. 83, pp. 982-996, Jun. 1995. 


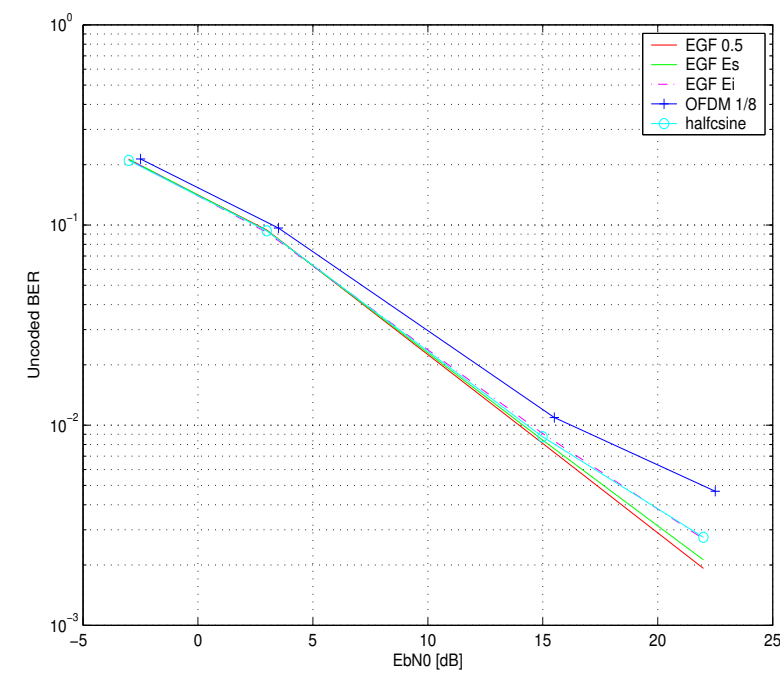

Figure 4: BER vs. SNR over channel B with $F=15 k H z$ for OFDM $\left(N_{c p}=\frac{N}{8}\right)$ and OFDM/OQAM with EGF (4 taps) and halfcosine (1 taps), 4QAM.

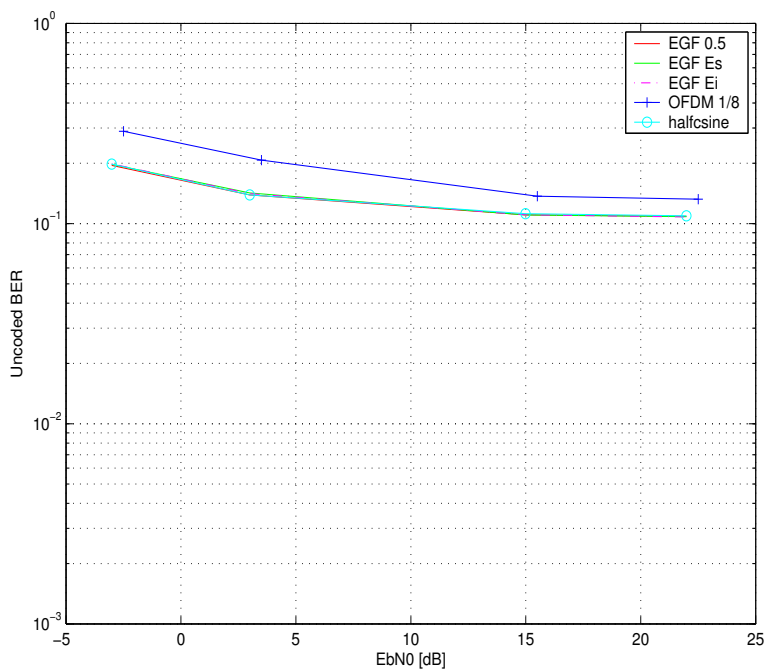

Figure 5: BER vs. SNR over channel $C$ with $F=15 k H z$ for OFDM $\left(N_{c p}=\frac{N}{8}\right)$ and OFDM/OQAM with EGF (4 taps) and halfcosine (1 taps), 4QAM.
[3] P. Jung, G. Wunder and C. S. Wang, "OQAM/IOTA Downlink Air Interface for UMTS HSDPA Evolution," 9th International OFDM-Workshop, Hamburg, pp. 153-157, 2004.

[4] M. Bellec and P. Pirat, "OQAM performances and complexity," IEEE P802.22 Wireless Regional Area Network, Jan. 2006.

[5] TIA Committee TR-8.5, "Wideband Air Interface Isotropic Orthogonal Transform Algorithm (IOTA) -Public Safety Wideband Data Standards Project Digital Radio Technical Standards," TIA-902.BBAB (Physical Layer Specification, Mar. 2003) and TIA-902.BBAD (Radio Channel Coding (CHC) Specification, Aug. 2003)

[6] P. Siohan and C. Roche, "Cosine-Modulated Filterbanks Based on Extended Gaussian Function," IEEE Transactions on Signal Processing, vol. 48, no. 11, pp. 3052-3061, Nov. 2000.

[7] J. Du and S. Signell, "Time Frequency Localization of Pulse Shaping Filters in OFDM/OQAM Systems," ICICS, Singapore, Dec. 2007.

[8] P. Siohan, C. Siclet and N. Lacaille, "Analysis and Design of OFDM/OQAM Systems Based on Filterbank Theory," IEEE Transactions on Signal Processing, vol. 50, no. 5, pp. 1170-1183, May 2002.

[9] F.-M. Han and X.-D. Zhang, "Hexagonal Multicarrier Modulation: A Robust Transmission Scheme for Time-Frequency Dispersive Channels,", IEEE Transactions on Signal Processing, vol. 55, no. 5, May 2007.

[10] G. Matz, D. Schafhuber, K. Gröchenig, M. Hartmann, and F. Hlawatsch, "Analysis, optimization, and implementation of low-interference wireless multicarrier systems," IEEE Transactions on Wireless Communications, vol. 6, no. 5, pp. 1921-1931, May 2007.

[11] W. Kozek, A.F. Molisch, "Nonorthogonal pulseshapes for multicarrier communications in doubly dispersive channels," IEEE Journal on Selected Areas in Communications, vol. 16, no. 8, pp. 1579-1589, Oct. 1998.

[12] T. Pollet, M. van Bladel, and M. Moeneclaey, "BER Sensitivity of OFDM Systems to Carrier Frequency Offset and Wiener Phase Noise," IEEE Transactions on Communications, vol. 43, no. 2/3/4, pp. 191-193, Apr. 1995.

[13] S. Signell and J. Huang, "A Simulation Environment for Multi-Antenna Software Defined Radio," in Proc. of ICICS07, Singapore, Dec. 2007.

[14] P. Hoeher, "A Statistical Discrete-Time Model for the WSSUS Multipath Channel," IEEE Transactions on Vechicular Technology, vol. 41, no. 4, pp. 461-468, Nov. 1992. 